\begin{tabular}{ll} 
Balkanologie & Balkanologie \\
\cline { 1 - 2 } & Revue d'études pluridisciplinaires
\end{tabular}

Vol. VI, $n^{\circ}$ 1-2 | 2002

Volume VI Numéro 1-2

\title{
Les géographes français et la délimitation des frontières de la Bulgarie à la conférence de la paix en 1919
}

French geographers and the demarcation of Bulgaria's borders at the 1919 Peace Conference

\section{Taline Ter Minassian}

\section{(2) OpenEdition Journals}

Édition électronique

URL : http://journals.openedition.org/balkanologie/454

DOI : 10.4000/balkanologie.454

ISSN : 1965-0582

\section{Éditeur}

Association française d'études sur les Balkans (Afebalk)

\section{Édition imprimée}

Date de publication : 1 décembre 2002

Pagination : 199-212

ISSN : 1279-7952

Référence électronique

Taline Ter Minassian, «Les géographes français et la délimitation des frontières de la Bulgarie à la conférence de la paix en $1919 »$, Balkanologie [En ligne], Vol. VI, n 1-2 | 2002, mis en ligne le 04 février 2009, consulté le 17 décembre 2020. URL : http://journals.openedition.org/balkanologie/454 ; DOI : https://doi.org/10.4000/balkanologie.454 


\section{LES GEOGRAPHES FRANÇAIS ET LA DÉLIMITATION DES FRONTIĖRES DE LA BULGARIE À LA CONFÉRENCE DE LA PAIX EN 1919}

Taline Ter Minassian*

\section{INTRODUCTION}

Inspirée d'une publication précédente ${ }^{1}$ consacrée au rôle des géographes français dans la délimitation des frontières balkaniques en 1919, et en particulier à l'action diplomatique et politique menée en faveur de la Roumanie par l'un des maîtres de l'école vidalienne, Emmanuel de Martonne ${ }^{2}$, cette recherche s'inscrit dans un champ récent de l'historiographie ${ }^{3}$ des relations internationales. Au lendemain de la Première Guerre mondiale, la contribution des géographes français à l'élaboration des frontières de l'Europe nouvelle témoigne en effet du lien existant dès cette époque entre savoir géographique et expertise. Le tracé des frontières de la Roumanie nouvelle et dans une moindre mesure de la Yougoslavie en sont de bons exemples, mais il s'agit d'Etats vainqueurs dont les revendications territoriales ont été satisfaites. Du côté des vaincus, comme la Bulgarie par exemple, les fondements géographiques des frontières de 1919 se sont trouvés inscrits dans la longue durée du $\mathrm{XX}^{\mathrm{e}}$ siècle, nourrissant le révisionnisme bulgare lui-même déterminant dans le choix des

\footnotetext{
* Maître de conférences à l'Université Jean Monnet (Saint-Etienne).

${ }^{1}$ Ter Minassian (Taline), « Les géographes français et la délimitation des frontières balkaniques à la Conférence de la paix en 1919 », Revue d'histoire moderne et contemporaine, 44 (2), avril-juin 1997, pp. 252286.

${ }^{2}$ Emmanuel de Martonne a inspiré depuis quelques années un grand nombre de publications parmi lesquelles il faut citer Bariety (Jacques), « Le Comité d'Etudes du Quai d'Orsay et les frontières de la Grande Roumanie, 1918-1919 », Revue Roumaine d'Histoire, 25,1996, pp. 43-51. Ce thème a également retenu l'attention des géographes, cf. Boulineau (Emmanuelle), « Un géographe traceur de frontières : Emmanuel de Martonne et la Roumanie », L'Espace Géographique, (4), 2001, pp. 358-369.
}

${ }^{3}$ Bariety (Jacques), « La Grande Guerre (1914-1919) et les géographes français », Relations Internationales, (109), printemps 2002, pp. 7-24. 
engagements de la Bulgarie pendant la Seconde Guerre mondiale. Les traités de Berlin et de Neuilly symbolisent ainsi jusqu'à aujourd'hui les humiliations subies par la nation bulgare comme en témoignent les études publiées en Bulgarie en 1999 à l'occasion du $80^{\mathrm{e}}$ anniversaire du Traité de Neuilly ${ }^{4}$.

L'étude des expertises géographiques et de leur incidence supposée ou réelle sur la définition des frontières des Etats de la péninsule balkanique au lendemain de la Première Guerre mondiale pose au moins trois problèmes. Servant "d'abord à faire la guerre", la géographie a contribué à formuler les conclusions territoriales d'une paix qui portait en germe, dans les Balkans comme en Europe occidentale, les affrontements futurs. Par ailleurs, l'action des géographes en tant que "traceurs de frontières" renvoie à une question d'ordre épistémologique propre à l'histoire de la géographie contemporaine. Les formes d'engagement des géographes pendant la Première Guerre mondiale ont permis ainsi de préciser l'image du géographe comme homme d'action. Enfin, dans une perspective de relations internationales, l'action des géographes pose le problème du poids hypothétique de l'expertise dans la "prise de décision" diplomatique.

Dans le cadre d'une telle problématique, l'étude de l'élaboration des frontières bulgares lors de la Conférence de la paix comporte évidemment plusieurs limites, puisque les sources des revendications territoriales de la nation bulgare remontent à Berlin (1878) et à la conclusion provisoire apportée à la fin des guerres balkaniques par le traité de Bucarest en 1913. Dans le contexte balkanique de la "Guerre des Sept ans" (1912-1918), le traité de Bucarest a été déterminant dans l'engagement de la Bulgarie aux côtés des puissances centrales. Vaincue en 1918, la Bulgarie n'a donc pas grand chose à attendre de la Conférence de la paix ce qui relativise d'autant, dans le cas bulgare, la pertinence de l'expertise géographique et son rôle dans l'élaboration des frontières.

Recrutés dans le milieu universitaire, les experts consultés lors de la Conférence de la paix constituent un groupe limité, majoritairement formé de géographes. Parmi les membres du Comité d'Etudes formé en 1917, seuls quelques uns ont été effectivement consultés par le Ministère des Affaires étrangères fournissant notes et rapports susceptibles d'influer dans le processus de prise de décision. Si Emmanuel de Martonne joue un rôle prééminent d'intermédiaire entre le Comité d'Etudes et la Conférence de la paix, d'autres universitaires, surtout des géographes, ont fourni des rapports sur les questions territoriales les plus complexes ${ }^{5}$ soulevées lors des négociations. Parmi

\footnotetext{
${ }^{4}$ N'oijski Dogovor, s objasnitelni belezki ot B.Kesjakov i Dim. Nikolov, Sofia : Martilen, 1999.

${ }^{5}$ BIF, Ms 4543, PV Comité d'Etudes, 2 avril 1919. Questions ayant appelé une expertise : frontière de la Transylvanie, Banat, frontière serbo-bulgare, frontière de la Drave, revendications belges, question du Slesvig, frontière orientale de la Pologne. Experts consultés : Demangeon, Denis, Haumant, de Martonne, Verrier, Gallois.
} 
eux, seuls quelques uns sont intervenus à l'échelon supérieur des négociations, comme Emmanuel de Martonne et Emile Haumant qui furent convoqués à plusieurs séances des commissions interalliées et des sous-commissions qui leur étaient rattachées.

\section{UNE OBSESSION RÉCURRENTE : L'INFLUENCE DE LA « BULGAROPHILIE AMÉRICAINE » AU SEIN DE L'INQUIRY}

Dans le corpus des sources disponibles - notes et rapports au Ministère des Affaires étrangères, procès-verbaux des réunions du Comité d'Etudes - les observations d'Emmanuel de Martonne relatives à la Bulgarie sont relativement rares, si on compare leurs occurrences aux nombreux témoignages de son obsession roumaine. Auteur d'un rapport sur les experts américains de YInquiry, Emmanuel de Martonne s'inquiète surtout du climat "bulgarophile" et "austrophile" régnant au sein de l'Inquiry dirigée par le Colonel House ${ }^{6}$, du moins à ses débuts.

\section{Les tendances initiales de l'Inquiry à l'égard de la Bulgarie}

La diplomatie française procède en effet à une évaluation des "buts de paix" américains et à un état des lieux des expertises et des influences entourant le président Wilson :

Depuis un an de nombreux câbles envoyés de Washington par le Haut-Commissariat ont détaillé les influences allemandes qui se sont exercées pour brouiller les titres français à un retour pur et simple de l'Alsace-Lorraine, les influences juives qui se sont exercées en faveur du maintien de l'Autriche-Hongrie, la tenace action du groupe de Cleveland H.Dodge et des ex-professeurs et élèves du Robert College à Constantinople pour empêcher toute rupture avec la Bulgarie ${ }^{7}$.

Envoyé en mission aux Etats-Unis (automne 1916) et auteur d'un rapport sur les membres de l'Inquiry, Emmanuel de Martonne déplore l'influence de Walter Lippmann sur les débuts de l'institution ${ }^{8}$. Il précise qu'« il faut tenir compte de certaines tendances pacifistes du début, de l'austrophilie et de la bulgarophilie de certains hommes connus : J.C.White et W.S.Monroe ». Le premier conseillait l'annexion de la Serbie et du Monténégro à l'Autriche, le se-

\footnotetext{
${ }^{6}$ House délègue la gestion des affaires de l'Inquiry à son beau-frère Sidney E. Mezes, président du City College de New York et philosophe des religions.

${ }^{7}$ MAE, A-Paix, 220, 15 décembre 1918, Note sur les travaux de l'Inquiry et les solutions de paix américaines par Louis Aubert, 11 décembre 1918, ff. 4-5.

${ }^{8}$ MAE, A-Paix, 220, Mémoire d'Emmanuel de Martonne sur le service américain de documentation (Inquiry), ff. 42-43.
} 
cond dépouillait la Grèce et la Serbie en faveur de la Bulgarie, demandait l'indépendance de la Macédoine et de l'Albanie et l'internationalisation de Salonique. Il semble ne rien comprendre au caractère changeant de l'ethnographie balkanique. On l'a reconnu récemment et ses mémoires sont considérés comme sans valeur. La bulgarophilie est néanmoins répandue aux EtatsUnis (importance du Robert College dont les 4/5 des élèves sont bulgares). Concernant la question de la Macédoine, il signale l'existence d'un mémoire déposé à l'Inquiry rédigé par un certain Sonnichsen ${ }^{9}$ " philobulgare, partisan d'une Macédoine indépendante et d'une Confédération balkanique dominée par la Bulgarie ». Sur la Dobroudja, le mémoire de W. S. Monroe était selon Emmanuel de Martonne «d'une bulgarophilie si accentuée qu'il a disparu des archives. On considère généralement que l'annexion par la Roumanie de la Dobroudja méridionale est injustifiée ». Concluant son rapport sur l'Inquiry, Emmanuel de Martonne juge que la documentation de l'lnquiry sur les Balkans est « insuffisante et partiale » en dépit de l'identité du rapporteur définitif, Clive Day, «pondéré et impartial ». Il attire particulièrement l'attention des diplomates sur la question de la Macédoine et sur le projet de Confédération balkanique en soulignant qu'on peut « prévoir une sympathie déguisée pour la Bulgarie ».

\section{Perception d'un réseau d'influence : diplomatie protestante et politique missionnaire}

Ces mises en garde répétées méritent d'être élucidées. Les nombreuses allusions aux réseaux d'influence actifs dans l'entourage de Wilson sont-elles exagérées ? Elles renvoient à l'influence de Cleveland H. Dodge - riche industriel américain et proche de Wilson - et à celle de la philanthropie protestante dont l'action humanitaire s'est exercée dans l'Empire Ottoman en faveur de la protection des Arméniens et des Bulgares. Cleveland H. Dodge est une figure centrale dans le réseau de la philanthropie protestante, fondateur du Dodge Relief Committee en faveur des Arméniens de l'Empire Ottoman (1915), membre incontournable de l'ACASR (American Committee for Armenian and Syrian Relief), proche de l'American Board of Commissioners for Foreign Missions. Issu d'une famille protestante presbytérienne impliquée de longue date dans l'action protestante au Proche-Orient, en particulier dans la fondation du Syrian Protestant College, Cleveland Dodge devient lui-même président du Board du Robert College en 1909. Ce lobby s'est révélé ex trêmement actif lorsqu'après l'entrée en guerre des Etats-Unis en avril 1917, s'est posée la question de la guerre ou de la

\footnotetext{
9 Journaliste américain, Albert Sonnichsen (1878-1931) fut un bulgarophile déclaré. Il a parcouru la Macédoine en 1906 en utilisant les réseaux clandestins de l'ORIM et a laissé un livre de souvenirs Sonnichsen (Albert), Confessions of a Macedonian bandit, New York : Duffield and Cie, 1909.
} 
neutralité à l'égard de l'Empire Ottoman et de la Bulgarie ${ }^{10}$. La perspective d'une entrée en guerre posait en effet la question de la protection des protestants de l'Empire Ottoman, des missionnaires et des responsables du Robert College. Les contacts existant entre Cleveland $\mathrm{H}$. Dodge, House et les missionnaires de l'American Board en Bulgarie dessinent les contours d'une diplomatie missionnaire qui a aidé Wilson à défendre, devant la Commission sénatoriale des affaires étrangères, le principe d'une non-intervention américaine contre l'Empire Ottoman et la Bulgarie. D'autre part, durant l'été 1918 le secrétaire de l'American Board, James L. Barton avait tenté une initiative afin de détacher la Bulgarie des puissances centrales ${ }^{11}$. Dans ce projet, Stepan Panaretoff, ambassadeur de Bulgarie aux Etats-Unis, devait jouer le rôle d'intermédiaire. Diplômé du Robert College où il a enseigné pendant près de quarante ans, proche de Cleveland H. Dodge et du jeune Washburn, Stepan Panaretoff a aidé les missionnaires à maintenir le principe de la neutralité américaine à l'égard de la Bulgarie. En juillet-août 1918, avec l'approbation de Stepan Panaretoff, James L. Barton projeta d'expédier Washburn en Bulgarie avec Edward C. Moore, trustee president de Y American Board, clergyman et professeur à Harvard. Sous la couverture officielle d'observateurs de la Croix Rouge, Washburn et Moore étaient censés offrir à la Bulgarie la médiation du président Wilson en faisant valoir les questions frontalières. Comptant sur le ressentiment des Bulgares brimés par les pertes territoriales de 1913, Barton et ses associés pensaient que la Bulgarie répondrait favorablement à une telle proposition. L'objectif de ce plan visait à couper la Turquie de l'Allemagne, forçant ainsi la Porte à s'incliner, voire même à ouvrir les Dardanelles à la flotte américaine. Ce projet fut effectivement pris en considération par House et par le State Department mais, à cette date, la perspective prévisible de la défaite de l'armée bulgare et les succès de l'expédition alliée en Macédoine poussant au nord de Salonique, rendaient déjà ce projet obsolète. L'ouvrage de Joseph L. Grabill consacré à la diplomatie protestante au Proche-Orient semble confirmer les remarques d'Emmanuel de Martonne sur l'influence du lobby protestant au sein de l'Inquiry où le sentiment de sympathie à l'égard de la Bulgarie était assez répandu. Cette influence du lobby protestant s'explique en particulier par le rôle de certaines institutions missionnaires américaines dans la formation des élites bulgares.

\section{Le rôle du Robert College dans la formation des élites bulgares}

Sans cesse mentionné dans les rapports d'Emmanuel de Martonne, le Robert College de Constantinople est une institution originale, de fait, la première institution américaine d'enseignement supérieur créée à l'étranger.

\footnotetext{
${ }^{10}$ Grabill (Joseph L), Protestant Diplomacy and the Near East, Missionary Influence on American Policy, 1810-1927, Minneapolis : University of Minnesota Press, 1971, p. 94 et suivantes.

${ }^{111}$ Ibid., p. 98.
} 
Fondé par les deux fils du révérend Harrison G. O. Dwight - missionnaire américain dans l'Empire ottoman - James H. et William B. Dwight, le Robert College doit son nom à celui de son principal bienfaiteur (mort en 1878), Christopher R. Robert, un riche homme d'affaires philanthrope et par ailleurs trésorier de l'American Home Mission Society. Au départ, l'objectif était de fonder un collège chrétien indépendant de l'American Board: Christopher Robert fit ainsi appel à Cyrus Hamlin qui quitta le Board pour se consacrer à cette nouvelle mission. Au moment de son ouverture en 1863, la structure du Robert College était tout à fait inhabituelle puisque les membres de la faculté (5) étaient plus nombreux que les étudiants (4) ${ }^{12}$. Fondé sur les principes d'une pédagogie missionnaire, l'enseignement au Robert College combine un cursus classique et technique (ouverture d'une école d'ingénieur en 1912). En 1868, le College s'installe sur son site définitif, au nord de Constantinople, offrant une vue splendide sur le Bosphore et la forteresse Rumeli Hissar. Proposant le même curriculum qu'un collège américain, le Robert College s'inspire du modèle des meilleurs établissements de la Nouvelle Angleterre, introduisant la pratique nouvelle du sport et combinant un enseignement en langue anglaise mais aussi dans treize autres langues. Gendre de Cyrus Hamlin, George Washburn (qui était venu à Constantinpole en 1858 en tant que trésorier local de l'American Board) devint son second président. Les principales sources de financement du Robert College ont été des philanthropes protestants de New York comme John Stewart Kennedy, William E. et Cleveland H. Dodge et bien sûr Christopher Robert qui fit don d'une somme totale comprise entre 400000 et 600000 dollars.

En quoi le Robert College est-il lié comme le suggère Emmanuel de Martonne au mouvement bulgarophile aux Etats-Unis ${ }^{13}$ ? Bien que l'influence du facteur russe et européen ait été déterminante dans le processus de l'indépendance bulgare, le rôle des missionnaires américains n'a pas été négligeable. Il faut rappeler en effet que la présence protestante a commencé en Bulgarie dès 1858 avec l'ouverture, à Samokov, du Collegiate and Theological Institute. Les presses de l'American Board ont contribué à l'édition de la plupart des livres imprimés en bulgare moderne, permettant ainsi le développement des relations culturelles entre les peuples slaves du sud-est de la Péninsule balkanique. D'autres signes témoignent encore des liens entre la présence protestante et le développement de l'émancipation nationale. Lorsque les protestants ouvrirent une Eglise évangélique à Sofia en 1888, le prince Ferdinand de Bulgarie assista à la cérémonie et fit don de $500 \mathrm{~F}$ à la congrégation. Mais surtout, jusqu'en 1890, les Bulgares formèrent en effet un effectif majoritaire

\footnotetext{
${ }^{12}$ Ibid, p. 23.

${ }^{13} \mathrm{Ibid}$, pp. 53-54
} 
parmi les diplômés du Robert College : sur 435 diplômés entre 1863 et 1903, près de la moitié étaient des Bulgares. Parmi eux, beaucoup se consacrèrent au service public en Bulgarie : en 1879, lors de la réunion de l'Assemblée constituante bulgare, les anciens étudiants du Robert College qui connaissaient la procédure parlementaire et les méthodes de gouvernement à l'occidentale prirent d'importantes responsabilités. Plus tard, de nombreux membres du Cabinet bulgare, des juges, des diplomates etc., sortiront encore du vivier du Robert College. Ainsi, le révérend George Washburn qui succèda à Cyrus Hamlin au poste de président du collège en 1877, fut considéré comme le "père de la Bulgarie" (tout comme Emmanuel de Martonne, "père de la Roumanie", quarante ans plus tard). George Washburn et Caleb F. Gates furent décorés par le roi de Bulgarie.

\section{IA QUESTION DES FRONTIÈRES BULGARES : UNE EXPERTISE « EN CREUX »}

En novembre 1920, dans un article publié dans les Annales de Géographie, Albert Demangeon n'hésite pas à écrire :

De tous les Etats danubiens et balkaniques, la Bulgarie est celui dont la constitution territoriale a le moins changé du fait de la guerre. Vaincue, elle n'a pas fait d'annexions comme la Grèce, la Serbie et la Roumanie ; mais elle n'a pas connu le démembrement, comme l'Autriche et la Hongrie. Elle demeure essentiellement ce qu'elle était, un Etat continental, ne s'ouvrant vers le dehors que par une mer intérieure et ne communiquant avec la Méditerranée que par des détroits qu'elle ne commande pas ${ }^{14}$.

En effet, le traité de Neuilly achève la fin d'un processus qui, du traité de Berlin au traité de Bucarest, a réduit à néant le projet de la Grande Bulgarie telle qu'elle s'était dessinée de manière éphémère à San Stefano.

Si pendant et après la guerre, au moment de la Conférence de la paix, les revendications territoriales bulgares ${ }^{15}$ sont à nouveau formulées, la Bulgarie vaincue ne peut nourrir l'espoir d'une conclusion territoriale qui lui soit favorable. Du côté des géographes et des universitaires, les rapports d'expertise

\footnotetext{
${ }^{14}$ Demangeon (Albert), «La Bulgarie », Annales de Géographie, (162), 15 novembre 1920, p. 401.

${ }^{15}$ Parmi une bibliographie pléthorique Bobtcheff (S.S.), Le peuple bulgare et ses aspirations dans le passé et le présent, Sofia : Imprimerie de la Cour Royale, 1915 ; Les accusations contre la Bulgarie, S.L.D., Délégation Bulgare à la Conférence de la Paix ; Piotitch (Stojan) (Balkanicus), The aspirations of Bulgaria, London, 1915 ; Die Bulgaren in ihren historischen, ethnographischen und politischen Grenzen, Berlin, 1917 ; Guechoff (I.E.), Tsokoff (D.), Mémoire adressé à la Conférence de la Paix, S.LN.D. , Ivanov (Jordan), Les Bulgares devant le Congrès de la Paix, Documents historiques, ethnographiques et diplomatiques avec 4 cartes, Berne : Librairie Académique, 1919 ; La question bulgare et les Etats balkaniques, Sofia, 1919 ; Reports and Letters of American Missionaries referring to the distribution of nationalities in the former provinces of European Turkey, 1858-1918, Sofia, 1919.
} 
évoquent "en creux" trois territoires, la Dobroudja, la Macédoine, la Thrace, dont l'attribution aux Etats vainqueurs des Balkans est quasiment une cause entendue. Dans ces conditions, on s'intéressera moins à l'efficacité de l'expertise dans la prise de décision qu'au type d'argumentation géographique dont chacun de ces territoires a été l'objet.

\section{La Dobroudja : l'argumentaire ethnogéographique}

Il n'est pas étonnant que la question de la Dobroudja soit l'objet des mentions les plus fréquentes dans les expertises fournies par Emmanuel de Martonne. Pour démontrer l'identité roumaine de la Dobroudja, le géographe évite curieusement de faire référence à la notion de région. Dans une monographie consacrée à la Dobroudja défendant le principe de l'attribution de celle-ci à la Roumanie, Emmanuel de Martonne préfère la notion, diversement connotée de "pays" ou de "terre": " S'il est une conclusion à tirer de l'étude ethnographique de la Dobroudja, c'est qu'elle n'a jamais été une terre bulgare et qu'elle tend naturellement à devenir une terre roumaine $»^{16}$. « Est-il vrai cependant que la Bulgarie ait des droits ethniques et historiques sur la Dobroudja supérieurs à ceux de la Roumanie ? C'est ce qui est beaucoup plus douteux $»^{17}$. Réfutant le principe d'une unité physique qui relierait la Dobroudja à la Bulgarie, l'argumentation d'Emmanuel de Martonne fait surtout appel - l'expertise frontalière mettant ici en évidence l'influence de l'épistémologie vidalienne - à la géographie humaine :

La géographie physique si elle mérite considération dans les problèmes politiques, semblerait au premier abord favorable aux revendications bulgares. Entre le Danube qui remonte vers le nord et la Mer Noire, les campagnes nues et sèches de la Dobroudja apparaissent comme la continuation des plateaux qui s'étalent au nord des Balkans, formés des mêmes couches crétacées non plissées, souvent recouvertes de tertiaire récent. Même absence d'eaux courantes sur de grandes étendues, mêmes villages très espacés. Pourtant l'aspect rappellerait plutôt encore les steppes russes que les plaines bulgares ${ }^{18}$.

L'exposé historique, remarquable par son érudition, tend ensuite à démontrer le lien pluri-séculaire qui aurait uni la Dobroudja à la Roumanie. La théorie des origines romaines est ainsi de nouveau mobilisée : " les Romains s'y sont établis en grand nombre, comme l'indiquent les ruines et les inscriptions. De même que la Dacie, la Dobroudja a été fortement romanisée $»^{19}$. Insistant sur les progrès économiques de la Dobroudja, roumaine depuis 1879 ,

\footnotetext{
${ }^{16}$ Martonne (Emmanuel de), «La Dobroudja », Rapport présenté à la séance du 6 mai 1918, in Travaux du Comité d'Etudes, Paris : Imprimerie Nationale, 1918, p. 10.

${ }^{17}$ Ibid.p.3.

${ }^{18}$ Ibid.

${ }^{19}$ Ibid.p.4.
} 
et sur la valeur stratégique de cette région qui assurait aux anciennes principautés moldo-valaques un débouché sur la Mer Noire, Emmanuel de Martonne fonde son argumentation sur des statistiques ethnographiques tirées, en l'absence de recensement digne de ce nom, de données partielles d'origine russe et roumaine concernant le département de Tulcea, situé dans la partie la plus septentrionale de la Dobroudja. Son commentaire de la carte ethnographique ainsi dressée démontre la "roumanité" de la Dobroudja :

Nous avons essayé de représenter plus exactement qu'on ne l'a fait jusqu'à présent la répartition géographique actuelle des races qui forment des groupes importants en Dobroudja, en laissant de côté les groupes sporadiques, où l'anthropologue retrouve des traces de presque toutes les races connues autour de la Mer Noire : Tziganes, Kurdes, Tcherkesses, Gagautzes. L'image reste celle d'un bariolage ethnique mais la prépondérance de l'élément roumain est évidente (...). Le mélange est extrême le long de l'ancienne frontière méridionale où on note des établissements bulgares assez récents, famille venues du Balcan de Kotel, ce qui indique chez les Bulgares comme chez les Roumains une tendance à la descente vers les plaines (...). Tout le long du Danube, la population est à peu près purement roumaine et, fait important que montre la carte, c'est là qu'est le peuplement le plus dense. Les régions ethnographiquement les plus mélangées ne sont pas les plus peuplées ${ }^{20}$.

Dans le PV de la séance du 6 mai 1918, au cours duquel Emmanuel de Martonne a présenté son rapport consacré à la Dobroudja ${ }^{21}$, il affirme de manière encore plus péremptoire que "rien ne justifie les revendications des Bulgares sur ce pays. Au moment de l'annexion à la Roumanie, les Bulgares n'y formaient pas le $1 / 5^{\mathrm{e}}$ de la population, d'ailleurs très clairsemée. Les Turcotatares y dominaient ». Et s'il reconnaît la présence bulgare, c'est pour en préciser immédiatement l'implantation récente : "Les collines du Babadag dans le nord restent le pays le plus mélangé et le seul où les Bulgares représentent un élément important. Ils y sont d'ailleurs des immigrés relativement récents, ce sont les colons transportés par les Russes en Bessarabie qui se sont arrêtés là sur le chemin du retour $»^{22}$. Il faut remarquer cependant - une fois assuré le principe du maintien des acquisitions roumaines en Dobroudja - une évaluation un peu plus objective sous la plume d'Albert Demangeon : «il fut un temps où la Bulgarie pouvait avec des apparences de raison prétendre à la Dobroudja » mais depuis que

la Roumanie a dû contre son gré, en 1878, prendre la Dobroudja en échange de la Bessarabie que gardait la Russie, il n'est pas contestable qu'elle a pris effectivement

\footnotetext{
${ }^{20} \mathrm{Ibid}, \mathrm{p} .10$.

${ }^{21}$ BIF, Ms 4543, PV de la réunion du Comité d'Etudes, 6 mai 1918, pp. 2-3.

${ }^{22}$ Ibid.
} 
possession du pays en le peuplant (en 1911, 54,7\% de Roumains, 14,3\% de Bulgares), en l'exploitant, en l'outillant; le port de Constantza, oeuvre roumaine, constitue un organe essentiel de la vie économique de la Roumanie. Par contre, ce sont uniquement des considérations stratégiques qui peuvent justifier la récente annexion de 1913 qui a donné la Silistrie aux Roumains ; car le pays n'est roumain ni par son économie, ni par son peuplement ${ }^{23}$

\section{Frontière occidentale de la Bulgarie et Macédoine : considérations stratégiques et géographie physique}

Concernant la frontière occidentale de la Bulgarie avec la Serbie, il est intéressant de constater que l'argumentaire géographique tourne à vide. Albert Demangeon reconnaît que ce sont des considérations stratégiques qui ont justifié la cession de quatre territoires à la Serbie ...

1) Au nord, le long du Timok, de la cession d'une portion de $130 \mathrm{~km}^{2}$ destinée selon Demangeon à "mettre en sécurité la voie ferrée de Zaietchar à Negotin $»^{24}$.

2) Dans le bassin de la Nichava, le long de la voie ferrée Nich-Sofia, de la cession du district de Tsaribrod «peuplé de 55000 habitants, tous Bulgares ».

3) A l'est de Vrania : la frontière est repoussée loin de la voie ferrée de Nich à Salonique.

4) Enfin, le saillant de Stroumitza « d'où les bandes bulgares attaquèrent souvent le chemin de fer de Salonique ».

... et en dressent ainsi le «bilan géographique » :

Ces pertes, toutes sensibles qu'elles soient, n'affaiblissent pas beaucoup la Bulgarie par elle-même. Mais les nouvelles frontières signifient bien davantage par ce qu'elles ne donnent pas à la Bulgarie, que par ce qu'elles lui enlèvent ; elles marquent que la Macédoine de Skopje, de Monastir et de Salonique, que les Bulgares considéraient depuis un demi-siècle comme un domaine de leur nation, leur échappe pour passer à la Serbie et à la Grèce ${ }^{25}$.

Concernant la Macédoine, les rapports et les discussions du Comité d'Etudes méritent d'être mentionnés. Celui de Haumant ${ }^{26}$ présente les arguments historiques des revendication bulgares et serbes (Empire bulgare, Empire serbe) sur la Macédoine, mais Charles Diehl fait valoir la «vanité des arguments historiques ». Haumant reconnaît qu'au XIX ${ }^{\mathrm{e}}$ siècle, « la propagande bulgare avait gagné la majorité de la population de la Macédoine ; mais

\footnotetext{
${ }^{23}$ Demangeon (Albert), art. cit., p. 402.

${ }^{24}$ Ibid., p. 403.

25 Ibid.

${ }^{26}$ BIF, Ms 4543, PV de la réunion du Comité d'Etudes, 8 juillet 1918, pp. 4-5.
} 
le ralliement à la Serbie s'était fait en 1913 sans difficulté, d'après le témoignage d'un Russe favorable aux Bulgares. Si la Serbie obtient un débouché sur l'Adriatique, il sera plus facile de solutionner la question de Macédoine, le pays étant incontestablement plus serbe au nord-ouest, plus bulgare au sud-est ». Même constat sous la plume de L. Gallois qui, faisant valoir le caractère indéfini des populations de Macédoine, affirme que « le principe des nationalités ne saurait ici être appliqué ${ }^{27}$. En conséquence, les géographes ont tendance à faire appel à une argumentation relevant de la géographie physique :

La vallée du Vardar est complètement séparée de la Bulgarie par des massifs de hautes montagnes et se rattache au contraire directement à la Serbie méridionale, formant l'extrémité de la grande voie de communications balkanique de Belgrade à Salonique.

A l'est du Vardar se trouve un massif de population turque compacte. C'est là que semblerait devoir être la vraie frontière.

Ce constat est assorti de considérations géopolitiques concernant l'importance de l'axe moravo-vardarien qui « ne doit pas tomber entre les mains des adversaires de l'Entente ». Emmanuel de Martonne souligne enfin qu'« il faut rappeler aux diplomates, qui sont assez portés à l'oublier, les réalités du relief et des grandes lignes de la géographie humaine, l'importance de la ligne Morava-Vardar ne saurait trop être mise en évidence ».

\section{La Thrace et la question du débouché méditerranéen : le prix de la défaite}

Cette question sensible entre toutes ne donne pas lieu à une argumentation géographique à proprement parler. On peut lire dans les procès-verbaux des réunions du Comité d'Etudes un rapport intransigeant de Pernot sur la question de la Macédoine orientale et de la Thrace affirmant nettement qu'« il n'y a pas lieu de laisser aux Bulgares un débouché sur la Méditerranée, réclamé seulement en vue d'ambitions politiques. Des facilités commerciales suffiront $»^{28}$. Contrairement au cas de la Dobroudja, l'argumentation ethnogéographique n'est pas utilisée : " nous ne possédons que des statistiques tendancieuses. Les grecques sont les moins mauvaises. Une enquête sur place permettrait seule de fixer la vérité ». Surtout, l'éventualité d'un débouché portuaire sur la Méditerranée est immédiatement subordonnée à des considérations politiques :

Cavalla revendiqué par les Bulgares, n'est pas le meilleur débouché de Sofia ; Salonique vaudrait au moins autant sinon mieux. Les marins estiment que Porto Lago pourrait faire un port plus abrité. On doit d'ailleurs tenir compte de l'état d'esprit des populations : Grecs et musulmans décimés par les déportations et la famine ont conçu contre

\footnotetext{
${ }^{27}$ Ibid, Complément au PV du 8 juillet 1918.

${ }^{28}$ BIF, Ms 4543, PV de la réunion du Comité d'Etudes, 21 février 1919, p. 2.
} 
les Bulgares une haine formidable. Ils accepteraient peut-être l'internationalisation, mais le rattachement à la Grèce serait bientôt réclamé. Autant y venir tout de suite, quitte à soumettre l'administration grecque à un contrôle ${ }^{29}$.

Emmanuel de Martonne se contente de demander des précisions sur les voies de communication entre Sofia et Salonique et sur les avantages que présenteraient Porto Lago ou Dédéagatch par rapport à Kavalla.

Il reste que ce parti pris ouvertement anti-bulgare suscite des critiques ouvertes au sein du Comité d'Etudes, notamment de la part du linguiste et orientaliste Antoine Meillet :

Les Grecs occupent depuis l'antiquité des rivages et seulement des rivages. Cela n'avait pas de gros inconvénients quand ils étaient la façade de populations barbares ; c'est intolérable devant des populations civilisées. La prétention des Grecs d'avoir tout le rivage de l'Egée sous prétexte qu'il y a relativement beaucoup de Grecs dans les ports ne saurait être soutenue. Je sais ce qu'on peut dire, et avec raison, contre les Bulgares. Il reste vrai qu'il sont la partie la plus active, la plus sérieuse, la plus laborieuse de la population des Balkans. Nous ne devons pas les mécontenter trop profondément, et justement, en leur refusant tout débouché sur la Mer Egée. En le faisant nous affaiblissons la position des Serbes vis à vis des Italiens, ce qui serait mauvais ${ }^{30}$.

$\mathrm{Ou}$ encore d'un certain Boyer qui, s'appuyant sur les témoignages des Lazaristes, des savants et des officiers de l'armée d'Orient, soutient que « les Bulgares sont un peuple de travailleurs, de bons administrateurs », argument également utilisé, il est vrai dans un autre sens, par ses contradicteurs qui reconnaissent dans les qualités des Bulgares, celles des Allemands : discipline, organisation matérielle, force. Dès lors, le "débat" sur les frontières ne fait pas tant intervenir la géographie que des stéréotypes faisant appel à l'idée de "civilisation" dont le développement de l'instruction apparaît comme le principal critère. Un défenseur de la nation bulgare au sein du Comité d'Etudes fait ainsi valoir la faible proportion d'illettrisme dans l'armée bulgare qui serait « moins grande qu'en France, alors qu'elle serait de $50 \%$ en Serbie, de $70 \%$ en Roumanie » (ce chiffre étant évidemment contesté par Emmanuel de Martonne). Très philhéllène, Pernot répond sans sourciller qu'il ne croit pas que « la disparition des illettrés prouve autre chose qu'un caporalisme scolaire à la prussienne » ${ }^{31}$ ! Quant au géographe Brunhes, il réfute la valeur du témoignage des Lazaristes sur les qualités du peuple bulgare : promoteurs du mouvement "Bolgaria", ils ont cherché à diviser l'Eglise orthodoxe dans l'intérêt du catholicisme ${ }^{32}$.

\footnotetext{
29 Ibid.

${ }^{30}$ Ibid.

${ }^{31}$ Ibid.

${ }^{32} \mathrm{Ibid}$, p.3. Sur la diplomatie missionnaire du côté catholique, voir le récent ouvrage de Fleury (Alain), Un collège français en Bulgarie, St Augustin, Plovdiv, 1884-1948, Paris : L'Harmattan, 2001, pp. 112-116.
} 
Ces discussions n'eurent probablement aucune incidence politique, mais elles révèlent sous un jour inhabituel, l'état d'esprit dominant au sein de l'intelligentsia française et du groupe des décideurs à l'égard du sort de la Bulgarie à la fin de la Première Guerre mondiale. Le traité de Neuilly comblera amplement leurs attentes en refusant à la Bulgarie tout accès à l'Egée. Pour Albert Demangeon, le traité de Neuilly « achève pour la Bulgarie l'œuvre du traité de Bucarest qui en laissant Kavalla aux Grecs, avait interdit à la Bulgarie de l'ouest de déboucher à la mer Egée par la vallée de la Strouma. La Bulgarie ne possède donc plus de communications avec cette mer qu'à travers le territoire grec. Le maintien du caractère continental et la fermeture de tout accès direct à la Méditerranée nous apparaissent donc comme les conséquences les plus graves de la guerre pour l'économie bulgare $»^{33}$.

\section{CONCLUSION}

Dans son article publié en 1920, consacré au tracé des frontières nouvelles de la Bulgarie et à leurs conséquences, Albert Demangeon cherche à justifier l'œuvre du traité de Neuilly en mettant en évidence les avantages géographiques d'une Bulgarie continentale : " c'est un avantage certain pour la Bulgarie, Etat continental, de grouper ses territoires en un bloc compact; elle peut y mieux ordonner sa circulation intérieure et mieux concentrer son organisation nationale $»^{34}$. Quels ont été les principes qui ont présidé à la définition des frontières bulgares ? Selon la remarquable typologie présentée par Stephen B. Jones ${ }^{35}$, il est clair que les frontières balkaniques de 1919 appartiennent au type anthropogéographique, fondées essentiellement sur un critère ethno-linguistique par excellence flou étant donnée la fiabilité relative de la plupart des cartes et des recensements. D'autre part, ce critère a été visiblement appliqué de manière parcellaire, de façon subjective, souvent lorsqu'il s'agissait de renforcer l'armature territoriale des Etats "gagnants" (cas de la Grande Roumanie). Concernant les Bulgares dispersés à l'extérieur des frontières en Macédoine, en Thrace, en Dobroudja ${ }^{36}$, et les allogènes de Bulgarie, on compte beaucoup sur l'assimilation. Peut-être faut-il percevoir ici un écho de

\footnotetext{
33 Demangeon (Albert), art. cit., p. 403.

${ }^{34}$ Ibid, p. 404.

35 Jones (Stephen B.), Boundary-Making, A Handbook for Statesmen, Treaty Editors and Boundary Commissioners, Washington DC : Carnegie Endowment for International Peace, 1945.

${ }^{36}$ Demangeon (Albert), art. cit., p. 413.
} 
certaines théories anglo-saxonnes de l'époque ${ }^{37}$, selon lesquelles il est souhaitable de faire passer les frontières au travers de régions densément peuplées, afin de promouvoir l'assimilation. Dans le cas d'un Etat vaincu comme la Bulgarie, où selon la propre estimation d'Albert Demangeon, « le déficit national de l'Etat bulgare » s'élève à 1200 000, soit plus du cinquième des Bulgares vivant à l'extérieur des frontières ${ }^{38}$, c'est un critère bien plus politique qu'anthropogéographique qui a déterminé le choix des tracés. La question du débouché maritime et le thème de la "prison continentale" bulgare est également prise en compte par Albert Demangeon ${ }^{39}$ qui reconnaît que la structure territoriale de la Bulgarie présente avec l'absence d'un débouché direct à la mer « une grave imperfection ». Ceci était susceptible d'alimenter l'irrédentisme bulgare et une aspiration à l'achèvement de l'unité nationale qui ne se réalisera qu'avec l'annexion temporaire de la Macédoine et de la Thrace de juin 1941 à octobre 1944.

${ }^{37}$ En particulier, Lyde (L.W.), Some Frontiers of tomorrow : an aspiration for Europe, London, 1915.

38 Demangeon (Albert), art. cit., p. 413. Avec beaucoup de précautions, Albert Demangeon évalue à 150000 le nombre des Bulgares de la « Nouvelle Dobroudja » pour 6400 Roumains, de 200 à 250000 le nombre des Bulgares de Thrace, à 800000 les Bulgares de Macédoine.

${ }^{39}$ Ibid, p.416. 